\title{
Role of Negative Stain Electron Microsocpy in the Diagnostics of Human Group C Rotavirus
}

\author{
C. D. Humphrey ${ }^{*}$, M. Metcalfe ${ }^{*}$, K. B. Clark ${ }^{* *}$, S. Lin $^{* * *}$, K. Foytich ${ }^{* *}$, and B. Jiang ${ }^{* *}$ \\ *Infectious Disease Pathology Branch, Division of Viral and Rickettsial Diseases, NCEZID, \\ ** Division of Viral Diseases, NCIRD, ${ }^{* * *}$ Division of Scientific Resources, NCPDCID, CDC, Atlanta, \\ GA 30333
}

Rotaviruses are classified into seven antigenic groups (A-G) based upon the distinctiveness of group antigenic viral protein 6 (VP6) (Fig.1) [1]. Rotavirus infection of the gastrointestinal tract is the leading cause of acute gastroenteritis in humans and animals worldwide. Vaccines against Group A rotavirus (GpA RV) may reduce prevalence of this major cause of severe diarrhea in humans. Two licensed rotavirus vaccines against GpA RV have shown 85\%-98\% efficacy in prevalence trials conducted in the Americas and Europe. However, prevalence studies may be impacted by malnutrition of subjects within the study population, or concurrent infections caused by bacteria, protozoan parasites, or unusual rotaviruses such as GpB and GpC RV. Attempts have been made to determine the disease prevalence of $\mathrm{GpB}$ and $\mathrm{GpC} \mathrm{RV}$ in the United States and Asia [2,3] but results of these studies have been negatively impacted by the lack of adequate diagnostic tools. GpC $\mathrm{RV}$ is commonly identified by seroprevalence studies thereby indicating that those tested have had exposure to the virus [4]. However, detection of GpC RV in feces by polymerase chain reaction methods (PCR) has been inconsistent and low because of the instability of the virus particles. The particle instability results in severely altered capsid integrity (Fig. 2) which leads to damage or loss of genomic materials [2, 4]. Morphology (Figs. 1,2) without specific immunological detection is insufficient to distinguish among various rotaviruses seen in stool of infected persons. Therefore, there is a need for highly specific antibodies against human $\mathrm{GpB}$ and GpC RV to determine the impact of these viruses on the efficacy of rotavirus vaccines designed to control GpA RV.

Propagation of human GpC RV in cell culture has thus far been unsuccessful so specific GpC antibodies cannot be obtained from conventional cell culture antigen. Therefore we used a recombinant baculovirus system to express viral proteins VP2 of the human GpC RV strain ASP 88 and VP6 and VP7 of the GpC strain S-1 to form recombinant GpC virus-like particles (VLPs) (Fig. 3) [5] and to produce antibodies against these proteins (Figs. 3, 4) [6]. Negative stain (NS) electron microscopy (EM) was used to monitor progress during the process that produced reactive antibodies to human GpC RV (Figs. 3-5) [6]. The role of EM used to monitor progress during GpC RV VLPs and subsequent antibody production will be described in the presentation.

\section{References}

[1] B. Jiang, et al. J. Infect. Dis. 172 (1995) 45.

[2] M. Esona, et al. J. Clin. Virol. 42 (2008) 221.

[3] M. Rahman, et al. L. Clin. Microbiol. 43 (2005) 4460.

[4] I. Abid, et al. J. Clin. Virol. 38 (2007) 78.

[5] K. Clark, et al. Virol. 387 (2009) 267. 


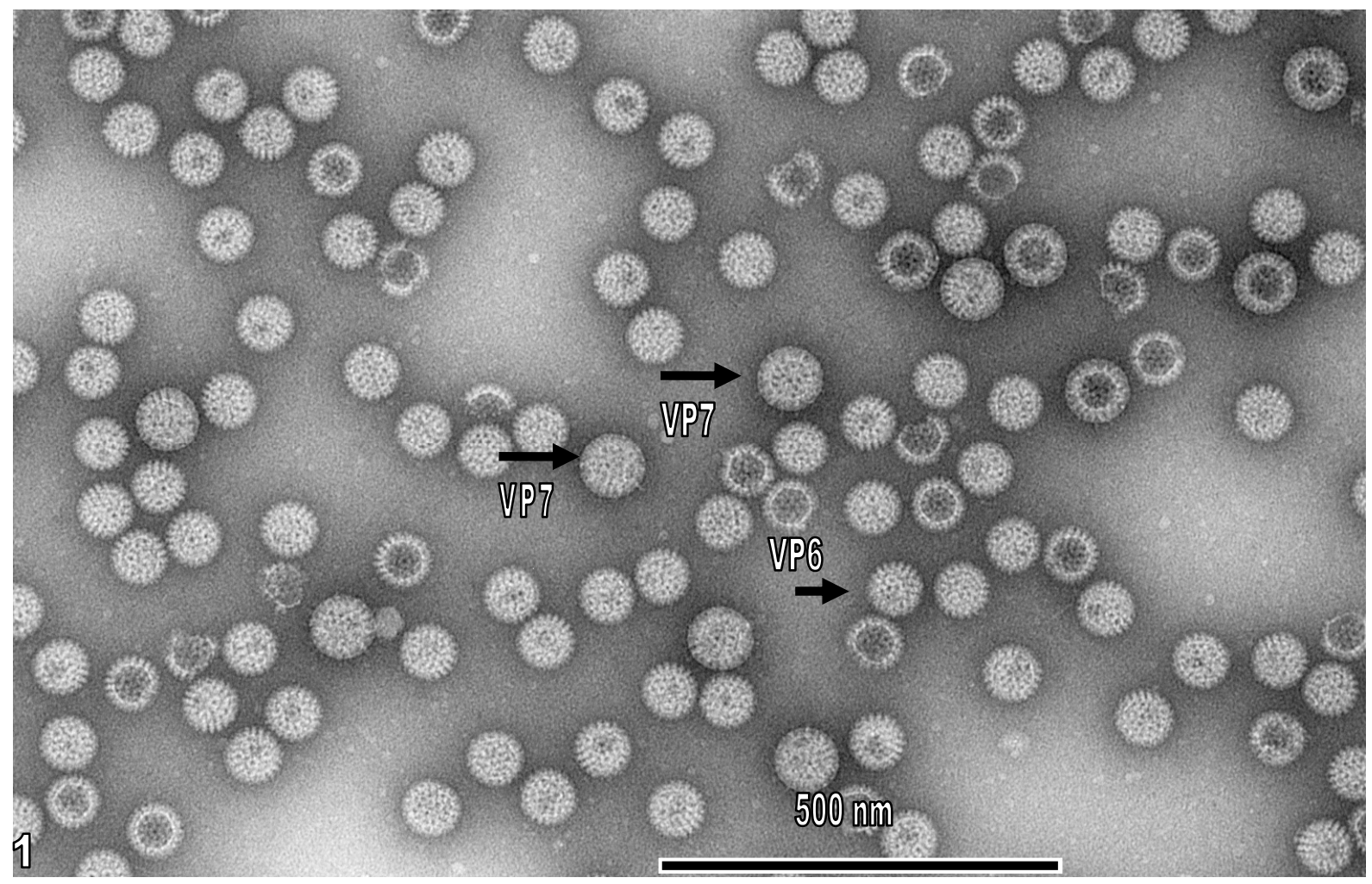

Figure 1. NS electron image of GpA rotavirus isolated in conventional cell culture. Viruses with all proteins including VP7 and those with VP6 exposed are present. 5\% ammonium molybdate-1\% trehalose NS was used in the preparation for this image and the images below.
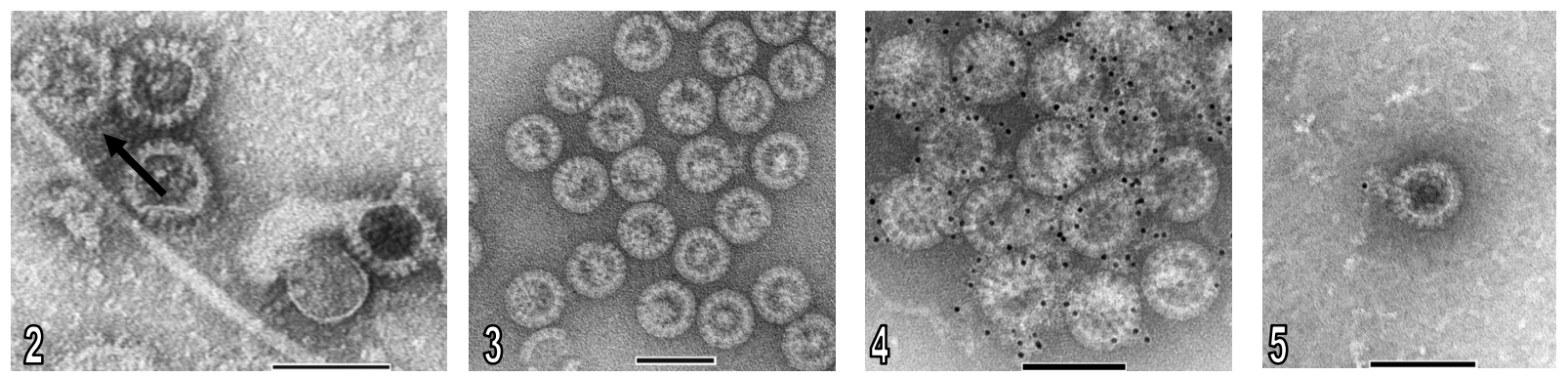

Figure 2. NS electron image of $\mathrm{GpC}$ rotavirus in human stool. Compare structure of damaged particle (arrow) with GpA rotavirus particles in figure 1 . Bar $=100 \mathrm{~nm}$.

Figure 3. NS electron image of recombinantly produced human GpC rotavirus VLPs formed of virus proteins 2, 6, and 7. Bar $=100 \mathrm{~nm}$

Figure 4. NS electron image of human GpC rotavirus VLPs labeled with human GpC specific rabbit hyperimmune serum decorated with $6 \mathrm{~nm}$ colloidal gold conjugated to goat anti-rabbit IgG.

Bar $=100 \mathrm{~nm}$.

Figure 5. NS electron image of GpA RV showing non-reactivity to human GpC specific rabbit hyperimmune serum. There is only a spurious $6 \mathrm{~nm}$ gold particle adjacent to a single $\mathrm{RV}$ and no virus-antibody aggregation such as that shown in figure 4 . Bar $=100 \mathrm{~nm}$.

Disclaimer: The findings and conclusions in this report are those of the authors and do not necessarily represent the views of CDC 